\title{
A MULTIPURPOSE ROBOTIC VEHICLE FOR THE RESCUE OF PERSONS AND INTERVENTIONS IN EMERGENCY SITUATIONS
}

\begin{abstract}
At present, multipurpose robotic vehicles represent the best possible solution for the rescue and transport of persons in a state of trauma from places in difficult-to-access terrain, in poor weather conditions and in emergency situations. It is a case of complementary means supplementing the equipment for rescue and emergency response that already exists or is being developed on the basis of a concept drawn in the framework of Integrated Emergency System of the Czech Republic. As a multipurpose means, the vehicle can be used for unmanned remote investigations into situations, for unmanned rescue and materials transport. Simultaneously, a modification to vehicles for places accessible merely by rail is designed.
\end{abstract}

Keywords: Population protection, emergency situation, robotic vehicle, vehicle; rescue of persons

\section{Introduction}

In the case of an incident, the response time of emergency response teams and the provision of medical assistance are a critical moment. Interventions in extreme areas and rough terrains require a specific approach [1], which is not usually possible by means of common emergency response vehicles. On the other hand, all-terrain vehicles for the transport of emergency response teams, required materials and equipment can accelerate the deployment of the teams in the course of intervention and the timely beginning of medical or other assistance. Subsequently, they enable the evacuation of injured and other persons. All-terrain emergency response vehicles seem to be an optimal solution for performing special tasks [2] during natural disasters, fires, interventions in inaccessible areas, during chemical and biological attacks, accidents, plane crashes and other accidents. They can also be used for the transport of hazardous materials and co-ordination of intervention actions.

\section{Materials and methods}

In the following part of the article, existing vehicles used in the rescue of persons and interventions in Europe are presented. These vehicles are divided into three groups, namely all-terrain vehicles, skid units for utility terrain vehicles and robotic vehicles.

\subsection{All-terrain vehicles for the rescue of persons and interventions}

Rescue and fire brigades in European countries use various models of all-terrain vehicles, four wheelers and snowmobiles.
A Czech initiative in the area of development of a terrain vehicle for rescue purposes was the utilization of an amphibious armoured personnel carrier OT-64 SKOT [3] on an $8 \times 8$ chassis, the prototype of which was constructed in the year 1959 for the needs of mechanized, communications and other special military units. An ambulance version of the vehicle without armament was designated OT-64 ZDRAV. For this ambulance version, a turretless version of the carrier was used and internal equipment was then adapted to the work of doctors. Inside 5 sitting patients or 2 patients lying on the stretchers on a special carry frame could be transported. In its time, the carrier was characterised by a very good design, fighting and driving properties, e.g. all eight-wheel drive, front and rear axle differential lock, two steerable front axles, central tyre inflation, planetary gearbox Praga Wilson, and others. The vehicle was driven by an air cooled diesel engine Tatra, which ensured trouble-free off-road and on-road driving. On a road the carrier was able to achieve the speed of about $100 \mathrm{~km} / \mathrm{h}$.

Simultaneously, it is necessary to state that these vehicles cannot be used for transport and work in rough terrains; the parameters of them do not make it possible to utilize required technologies and emergency response equipment for emergency situations, do not connect the advantages of a robotic system with those of a manoperated system, and are too robust, without advanced technologies that correspond to the existing results of research and development in the area of equipment and technologies for rescue and emergency response.

At present, some fire brigades use firefighting and rescue sixwheelers mounted on a known all-terrain Polaris Ranger $6 \times 6$ chassis, from Rosenbauer, an Austrian manufacturer of fire appliances [4]. With the Polaris firefighting and rescue vehicles, includ-

\footnotetext{
* David Rehak, Ales Dudacek, Pavel Polednak

Faculty of Safety Engineering, VSB - Technical University of Ostrava, Czech Republic, E-mail: david.rehak@vsb.cz
} 
ing the $4 \times 4$ variant, many fire and rescue services all over the world are equipped. The vehicles are used in forest firefighting, interventions in confined spaces, in mountain terrain areas, etc. The range of purposes for which they are intended is thus relatively wide, namely from a first response vehicle to a special emergency vehicle. To the wide range of applications, modifiable equipment of them is adapted.

Other vehicles that are available also in the Czech Republic are the models of the Canadian company ARGO [5] that are designed, among other matters, for firefighters and search and rescue operations. A lightweight model ARGO Avenger $8 \times 8 \mathrm{EF}$ is designed for enabling the transport of a patient and other 3 persons. The vehicle can transport up to 6 passengers and has a carrying capacity of about $520 \mathrm{~kg}$, and a towing capacity of more than $800 \mathrm{~kg}$. For the use of the vehicle on snow and marshy land, tracks compatible with tyres can be used. In addition, the amphibious version of the vehicle is available too.

ARGO is a low-cost and easy-to-transport vehicle suitable for operations in the course of catastrophes. For emergency tasks, these vehicles have been utilized since the year 1990 and are able to overcome water, flooded areas, snow, ice, sand, stones and various other obstacles. For terrain interventions and for the rescue of persons, e.g. during natural disasters, models Centaur $954 \mathrm{G}$ (with a petrol engine or a turbodiesel engine), $8 \times 8$ Avenger $700,8 \times 8$ Frontier 650 and $6 \times 6$ Frontier 650 are intended.

The Swedish company BAE Systems [6] manufactures allterrain vehicles Hägglunds similar to a snowmobile. These tracked vehicles are used by fire services, rescue services and armed forced. An all-terrain vehicle Hägglund consists of two tracked units (with a reinforced GRB body) linked together by an articulated steering joint. The vehicle operates on snow and in mountain areas and is also able to operate under extreme conditions. Its normal operations include search and rescue operations, firefighting and, to a certain extent, working with hazardous materials. The front part of the vehicle was designed to be able to carry firefighting equipment, including two sets of breathing apparatus and spare bottles.

However, it is necessary to state that this system of articulated design is not able to operate in very hard-to-reach places in rough terrains; it does not provide a possibility of multipurpose utilization and operative changes in parameters (e.g. utilization in tunnel structures). It neither provides advantages of a robotic system nor enables any remote control.

\subsection{Skid units for emergency response vehicles}

In the United States of America, the company Kimtek [7] manufactures special skid units intended for mounting on bodies of common utility all-terrain vehicles. These units enable the presence of a rescuer with equipment and an assisting paramedic next to the patient during transport on the utility vehicle even in difficult-to-access terrain and from hard-to-get-at places. The universal application of these units is possible thanks to their design which is suitable to almost all utility all-terrain vehicles.

Medlite Transport (MTS-11) is designed to carry one patient, one emergency medical technician attendant or a paramedic and assorted emergency gear. The stretcher is easily attached to the transport unit by straps which are part of the unit. The seat for the attendant accompanying the patient is equipped with a seat belt and is able to move on wheels along the whole length of the unit and to be locked in three different positions. Under the area for the patient, there is a storage area for medical bags, auxiliary materials, oxygen, and others. Also the extended version Medlite Transport Deluxe (MTD-11) is manufactured, which is equipped, in addition to the above-mentioned items, with a slide-out tray, a holder for oxygen bottles and rails for the seat for the third person.

The disadvantage of this system is the fact that the special skid unit for utility vehicles is not linked to the mobility module with parameters of the multipurpose vehicle being designed; moreover, it does not enable the installation of a comprehensive set of resuscitation elements.

\subsection{Robotic vehicles for the rescue of persons and interventions}

Recently great attention has been paid to the development of robotic vehicles for the rescue of persons and interventions at the Technology Research Center (TATRC) [8], at which several important projects have been dealt with.

The project RCCEE [9], the objective of which was to develop a system being able to evacuate and transport injured soldiers from the place of injury to a field hospital, can be regarded as the most significant. A new vehicle, which was manufactured in the framework of the project, consists of a small mobile manipulator REX (Robotic Extraction Vehicles) that is utilized for the search for and the short-distance transport of a patient, and of a fast tracked vehicle REV (Robotic Evacuation Vehicle), which is used for the transport of a patient with supporting the vital functions of the patient over a longer distance (to a field hospital). The smaller REX is transported inside the larger one; the REV can transport up to two REXs. The task of the REV is to remain in the vicinity of an injured person and to help remote control the REX, which moves independently towards the injured person. The main task of the REX is to localize the patient and to transport him/her on the stretcher back to the REV. After dispatching the REX to another operated mission, the REV transports a patient to a field hospital and then returns back to the site of intervention. Transport thus functions in a loop.

Another significant project is the programme REMeD-D (Robotic Emergency Medicine and Danger-Detection) [10], the main objective of which is to develop technologies enabling emergency response actions in the terrains, where medical supplies are missing, for the safe and remotely controlled rescue of injured 
persons, e.g. also in situations involving threats of chemical and/or radiological contamination. The system consists of an evacuation robot with a stretcher and two robots equipped for the detection of chemical and radiological agents. The company Applied Perception is responsible for the development of the robot and operator software, electromechanical integration of patient extraction and robot loading and for an overall design proposal. Remote control is possible thanks to a revolving camera (with daylight and thermal images) and chemical and nuclear sensors for contaminated land investigation and for detection of potential victims and their transport to a safe place.

In conclusion we can state that nowadays in the Czech Republic and in the states of the European Union, such multipurpose lightweight vehicle is missing in the area of safety and rescue vehicles that could be used in several variants with operative changes in parameters and that would be designed both for the rescue of persons and the preservation of material values in emergency situations, during terrorist attacks, catastrophes and accidents, including incidents in railway tunnel structures, and as a multipurpose remote-controlled robotic vehicle or surveyor.

\section{Results}

In response to the unsatisfactory situation in European countries, the development of a multipurpose robotic vehicle (see Fig. 1), designed for the rescue of persons and interventions in emergency situations in rough terrain and climatic conditions, with a possible operative change in parameters, and also for the alternative utilization in railway tunnels, was commenced in the Czech Republic in the year 2009. Another alternative is its utilization as a robotic vehicle designed for investigations and interventions that is remotely controlled by an operator (possibility of unmanned rescue) and that can also be controlled by a driver. The vehicle is a mechatronic system of the robotic type [11], i.e. based on the integration of state-of-the-art robotic and emergency technologies. The alternatives of the transporter variants are defined by special equipment oriented on the utilization in specific emergency situations.
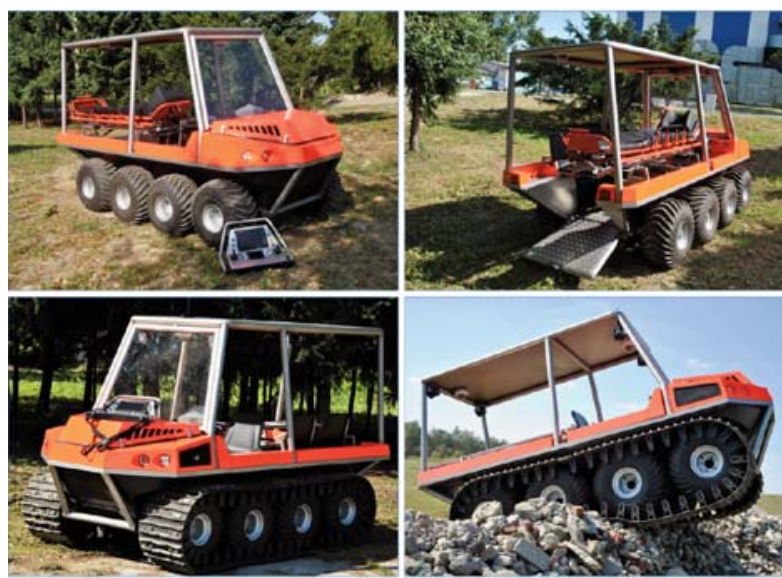

Fig. 1 Multipurpose robotic vehicle

\subsection{Description of robotic vehicle}

From the point of view of functions and use, a robotic vehicle is maximally universal. It is the case of a device that can be simply and easily transported without using demanding transport means - a container pulled on a carrier of the type used by the services of Integrated Emergency System of the Czech Republic. The vehicle is able to operate in rough terrain, on snow and marshy land, and is able to cross even rather small bodies of water. In addition to the function of controlled "all-terrain ambulance", the device is equipped with a remote control system, including a camera system; it is able to be used as a surveyor robot or, in case of danger to the crew, this device can be sent to assist the injured person and are controlled remotely. For the reason of extending the universality, a possibility of operative changes in parameters of the chassis for driving the rails were dealt with, which ensures the capability to intervene even in hardly accessible (mountain) railway tunnels [12]. The remote control of the vehicle makes possible to dispatch it to a place with minimal visibility (smoke, fire) and to address the injured person by means of voice transmission.

On the multipurpose robotic vehicle, the following basic requirements were put:

- total width of the vehicle of $1.8 \mathrm{~m}$;

- total length of the vehicle of $3.4 \mathrm{~m}$;

- low fuel consumption of $3.5 \mathrm{l} / \mathrm{h}$;

- loading capacity of $550 \mathrm{~kg}$;

- possibility of rapid grasping the stretcher for a patient, including ensuring the access of an attendant or paramedic;

- roof construction containing the frame that ensures the safety of the occupants in case of overturning the vehicle;

- detachable roofing protecting the occupants from unfavourable weather effects;

- overall concept and version of the vehicle with emphasis on simplicity, robustness and functionality;

- temperature range of operating environment from $-20{ }^{\circ} \mathrm{C}$ to $+50{ }^{\circ} \mathrm{C}$.

\subsection{Requirements for individual subsystems}

During the development of the multipurpose robotic transporter, high requirements were put on individual subsystems, i.e. a mobility subsystem, stretcher subsystem, interface subsystem, visual, communication and navigation subsystem, control subsystem, source subsystem and protection subsystem [13].

The mobility subsystem is designed for ensuring the high ability of the vehicle to pass across difficult terrain, to ford and to sail, the minimal climbing ability of $70 \%$, the ability to descend a 45 degree slope; all this at a small mass. The maximum speed reaches $32 \mathrm{~km} / \mathrm{h}$. The vehicle has good manoeuvrability (differential driving), a wheel chassis with a possibility of simple putting the tracks on, a low centre of gravity ensuring a high degree of stability and the minimal 2 hour operation time per one tank. The minimum chassis clearance is $150 \mathrm{~mm}$ at the used wheel diameter of $25^{\prime \prime}$ and the minimum height of an obstacle that the chassis is able to overcome without the tracks on, is $400 \mathrm{~mm}$. 
The stretcher subsystem (see Fig. 2) fulfils the requirements prescribed in a European standard [14]. For the safe conveyance of a patient in terrain, it is equipped with a multi-point restraint system. Last but not least, positioning the upper and the lower half of the patient's body within the standard required range is possible - however, positioning the patient is not possible in the course of off-road run.

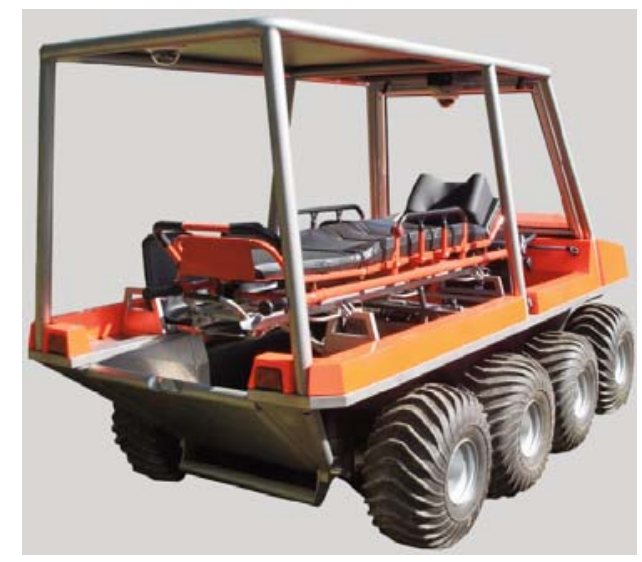

Fig. 2 Stretcher subsystem

The interface subsystem enables the stretcher system to be easily and quickly locked with and released from the frame of the multipurpose robotic vehicle. The interface was developed with regard to the unification of similar equipment used for the transport of stretchers in ambulances and air ambulances of rapid rescue service. Simultaneously it necessarily provides at least partial damping and absorption of shocks generated by the movement of the vehicle in rough terrain.

The visual, communication and navigation subsystem ensures communication between the vehicle and the operator in the emergency response vehicle or at the base (voice and image transmission). Above all, it is the image transmission from an environment in which the vehicle is just moving in good visibility conditions (front and back cameras with infrared lighting), image transmission in reduced visibility conditions (darkness, fog, smoke, etc.) and voice transmission from the operator's station to the crew of the vehicle. For route optimization and unmanned operation optimization, the real-time position (orientation) of the vehicle, the speed of its movement and the location determined by GPS is continuously determined. The future of this developmental trend is proved by the fact that autonomous 3D mapping systems to support the activities of emergency response teams have already been developed abroad [15].

The control subsystem enables the unmanned remote control of all functions of the universal robotic vehicle from the operator's station and is able to operate at least in restricted mode and for a limited time in case of a failure of the vehicle combustion engine. In addition to the superior control system, part of the control subsystem is also a system of control of the vehicle by the driver; it has an ergonomic arrangement of control elements and supports the simple and intuitive handling of the vehicle. With reference to the operating environment, the control elements of the universal robotic vehicle is sufficiently robust - main control elements for controlling the speed and the direction of running, in the case of running in terrain, enable the driver to sink into the seat without negative effects on the speed and/or the direction of running.

The basis of the source subsystem is a combustion engine (Kohler Aegis LH 775, liquid cooled two-cylinder four-stroke ignition engine), a properly dimensioned source of electrical energy (generator connected to the combustion engine), which covers the needs of the control and the other systems of the vehicle, and a reserve battery to ensure the emergency operation of the control system in the case of a failure of the combustion engine. The source subsystem enables the movement of the vehicle in difficult terrain, especially great tilts in the longitudinal and traverse directions (tilt in longitudinal direction is $50^{\circ}$, in transverse one $40^{\circ}$ ). All components of the source subsystem were selected with regard to the minimum mass, space to be occupied by them and high efficiency.

The protection subsystem consists in the mounting of detachable parts, which roof over and protect all occupants of the vehicle from unfavourable weather and climate effects, on the vehicle.

\subsection{Communication subsystem for control data transmission}

For the transmission of control commands for remote controlling the robotic vehicle, a system from the company RadioCrafts, namely the model RC1280HP [16] providing sufficiently great transmit output power are used. The module can be adjusted to transmit at the output power of up to $500 \mathrm{~mW}$, which is 20 times more than in the case of transmission in the WiFi band. Other advantages of this system are mainly small dimensions $(19.5 \times$ $60.5 \times 6.0 \mathrm{~mm}$ ), low consumption, multi-channel operation at a licence-free band of about $869 \mathrm{MHz}, 25 \mathrm{kHz}$ narrow band, 80 communication channels, range of up to $1 \mathrm{~km}(6 \mathrm{~km}$ if an antenna is used), easy setting by means of ASCII characters through a converter RS-232/485 and a converter the operation of which complies with the regulations of EU R\&TTE [17][18][19].

\subsection{Control subsystem}

The function of the control subsystem of the vehicle is to receive commands from the operator's station of the vehicle and, on the basis of them, to implement the control of the following subsystem components:

- Accelerator - a strand to the handlebar grip;

- Brake - electromagnetic with multiple laminated disk;

- Gearbox - control lever Forward / Neutral / Reverse;

- Gearbox - Hi / Low high or low gearing (change in moments);

- Driving - handlebar left, right (vehicle turns on the principle of braking of one side). 
The electronics of control of the above-mentioned movement either receives commands by means of wireless channel from the operator's station, or can be switched to manual control directly from the vehicle; the switch is implemented by a suitable control element. In the framework of simplicity and reliability, for receiving the commands from the operator's station, a full-functioned PC is not used in the vehicle but merely a suitable electronic single-chip module from the company Atmel [20] is used. This module provides the receipt of data, data interpretation and the distribution of commands to individual control members participating in the control of vehicle running.

The control subsystem contained in the control console of the operator (see Fig. 3 ) is equipped with a computer for the operation of vehicle navigation. The control console is equipped by compact control system enabling the transporter handling by joystick and its basic monitoring by image transfer into built screen.

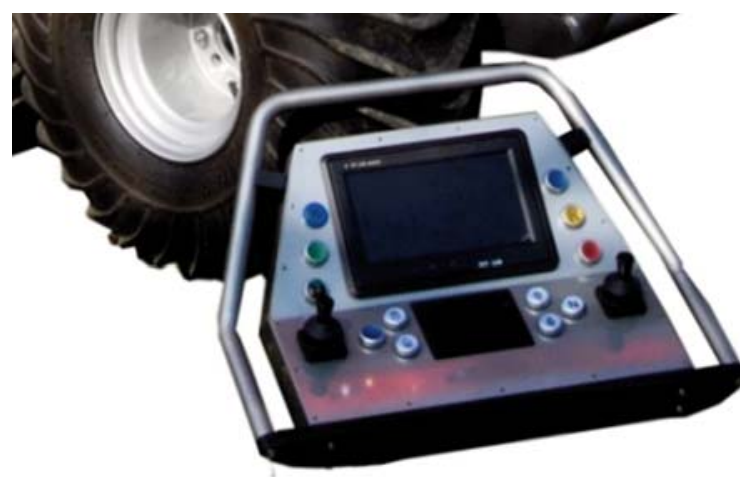

Fig. 3 Operator's console

\subsection{Visual subsystem}

An integral part of the control system of the remote-controlled vehicle is the visual subsystem ensuring the transmission of image information from the vehicle to the operator's station. This image information enables the remote control of the vehicle by the operator and also serves for evaluating the situation in the place of movement of the vehicle. As for the image transmission, two fundamental principles of image data transmission, namely digital and analog ones are considered.

In the case of digital image transmission, digital cameras with a USB, LAN or FireWire interface, which are connected to the control computer forming part of the remote-controlled vehicle, are used. The advantage is a possibility of software-assisted checking the rate of compression, and the topicality and correctness of transmitted data with regard to possible jamming the wireless communication channel. This conception makes it possible to transmit up to two data streams with images from two cameras independent of each other. However, the disadvantage of this solution is a necessity of using full-functioned computers with a Windows operating system (industrial as well as compact), which leads to several unfavourable effects, such as the long startup time of the whole system (up to several minutes in order), which is necessary for booting the system both on the transmitting side and on the receiving side. This is particularly disadvantageous to mobile applications, because a full functioned computer (which, in addition to this, executes the tasks of compression and decompression of realtime image data transmitted online) also requires an adequate power supply.

In the case of analog image transmission, a broad spectrum of cameras designated CCTV (closed-circuit television) [21] can be used. These analog cameras (more specifically digital cameras with analog output) are offered by many companies, which provide a possibility of selecting the camera that is the most suitable for the given purpose, i.e. from the point of view of mounting, coverage, sensitivity, etc. As an example, a camera Sony SSC-CD79P with an IP66 enclosure can be given [22]. The great advantage of this camera is its unbreakability, excellent housing, resistance to powerful waterjets, and its exceptional sensitivity even in poor light conditions.

\subsection{Navigation subsystem}

For orientation in terrain and positioning the remote-controlled vehicle in relation to the operator's station, the use of a system of global positioning is the most suitable. The placement of a receiver in the vehicle itself and a receiver in the operator's station is assumed. In a simpler variant (i.e. navigation without connecting to map systems), the above-mentioned configuration of GPS receivers enables the implementation of only limited possibilities of navigation. In the main, it is possible to determine the real distance between the vehicle and the operator's station and the world orientation from the place of control of the vehicle. Another functionality would be a possibility of storing the information on position. Data stored like that could be subsequently used for the inverse analysis of movement of the vehicle [23] and also the operator's station.

In a case of the integration of the navigation subsystem with a suitable map system, its possibilities would be considerably increased, e.g. representation of the position of the vehicle in the map base in real time [24]. For navigation, an external map system could be used (e.g. with the map base for the whole country, all Europe, other countries of the world) or a system when as a base is used an ortho-photo map (aerial photograph) of the given locality that after calibration in relation to the GPS coordinates are used as a base for the representation of the position of the vehicle in the given environment.

\section{Conclusion}

The presented multipurpose robotic vehicle is a device designed for the rescue of persons and interventions in emergency situations. It is the case of an all-terrain emergency response vehicle that rep- 
resents an optimal solution for providing assistance in natural disasters, fires, accidents, plane crashes and other interventions in inaccessible areas, in the course of chemical and biological attacks, and can be also used for the transport of hazardous materials and co-ordination of intervention actions. On the basis of integrated comparative analysis it can be stated that with such a vehicle no emergency team in the Czech Republic as well as in other states of the European Union is equipped at present.

The results of the project form the conditions for interventions in emergency situations, in conditions and localities which are practically inaccessible by means of the existing equipment. In this way, the level and the efficiency of intervention and also the safety of members of rescue and emergency response teams are influenced markedly. For this reason, services of not only the Integrated Emergency System of the Czech Republic but also abroad have shown interest in a multipurpose rescue and emergency response robotic vehicle conceived like that.

\section{Acknowledgments}

The paper has been elaborated within the project of the Ministry of Industry and Trade of the Czech Republic filed under FRTI1/572 and entitled "Research and Development of Multipurpose Robotic Transporter for Rescue of Persons and Intervention in Crisis Situations in Particularly Tough Terrain and/or Climatic Conditions".

\section{References}

[1] NARDI, C.D., FUMITOSHI, M., VOYLES, R.: Safety, Security, and Rescue Robotics. IEEE Robotics \& Automation Magazine, 2008, vol. 15, pp. 12-13, DOI: 10.1109/MRA.2008.921537.

[2] TADOKORO, S., ERKMEN, A., ERKMEN, I.: Rescue robotics special issue. IEEE Robotics \& Automation Magazine, 2002 , vol. 9, pp. 4-8, DOI: 10.1109/MRA.2002.1035205.

[3] History: Armoured Personnel Carrier OT-64 [online]. Ministry of Defence, 2012 [cit. 04-11-2011]. Available at: http://www.army.cz/scripts/detail.php?id=6042

[4] Rosenbauer [online]. Available at: http://www.rosenbauer.com/

[5] Argo [online]. Available at: http://www.argoatv.com/

[6] BAE Systems [online]. Available at: http://www.baesystems.com/index.htm

[7] Kimtek [online]. Available at: http://www.kimtekresearch.com/

[8] Telemedicine \& Advanced Technology Research Center [online]. Available at: http://www.tatrc.org/

[9] TATRC and TARDEC Collaborative Robots Program [online]. Telemedicine \& Advanced Technology Research Center, 2011 [cit. 12-12-2011]. Available at: http://www.tatrc.org/ports/robotics/docs/AUVSI_TATRC_TARDEC_Robot_Team.pdf

[10] Robotic Emergency Medicine and Danger-Detection [online]. Telemedicine \& Advanced Technology Research Center, 2011 [cit. 12 12-2011]. Available at: http://www.cermusa.francis.edu/ uploadedfiles/presentations/REMeD-DpresentationforATA5-1407 PRINTVERSION.pdf

[11] JURISICA, L.: Mechatronics Systems. Communications - Scientific Letters of the Universtiy of Zilina, 2007, Vol. 9, No. 1, pp. 5-7. ISSN 1335-4205.

[12] HUANG, S. L.: Measures on the Emergency Repairs on Bao-Cheng Railway Tunnel 109\#, In LIANG, W.H., LI, Q., GAO, B. (Eds.): Proc. of intern. conference on Earthquake Engineering - The first anniversary of Wenchuan earthquake, 2009 Chengdu. Southwest Jiaotong Univ Press, 2009, pp. 584-588.

[13] VIDA, J., CERNY, M., SEBEK, M.: Requirements Definition for Solving Individual Subsystems, In BARTOS, P., HARMECKO, A., DUDACEK, A. (Eds.): Research and Development of Multipurpose Robotic Transporter for Rescue of Persons and Intervention in Crisis Situations In Particularly Tough Terrain and/or Climatic Conditions (Research report). 1st ed. Ostrava, Czech Republic: FITE, 2011. (in Czech)

[14] European Standard EN 1865-1:2010. Patient handling equipment used in road ambulances - General stretcher systems and patient handling equipment.

[15] NAGATANI, K., et al. Multirobot Exploration for Search and Rescue Missions: A Report on Map Building in RoboCupRescue 2009. J. of Field Robotics, 2011, vol. 28, pp. 373-87, DOI: 10.1002/rob.20389.

[16] RadioCrafts, Product RC1280HP [online]. Available at: http://www.radiocrafts.com/index.php? sideID=278

[17] European Standard EN 300 220:2006. Electromagnetic compatibility and Radio spectrum Matters (ERM) - Short Range Devices (SRD) - Radio equipment to be used in the $25 \mathrm{MHz}$ to $1000 \mathrm{MHz}$ frequency range with power levels ranging up to $500 \mathrm{~mW}$.

[18] European Standard EN 301 489:2002. Electromagnetic compatibility and Radio spectrum Matters (ERM) - ElectroMagnetic Compatibility (EMC) standard for radio equipment and services.

[19] European Standard EN 60950:2000. Safety of information technology equipment.

[20] Atmel [online]. Available at: http://www2.atmel.com/

[21] SRIMUK, P., MITATHA, S., YUPAPIN, P. P.: Novel CCTV Security Camera System Using DWDM Wavelength Enhancement, In FUJII, Y. (Ed.): Proc. of 1st intern. conference on Security Camera Network, Privacy Protection and Community Safety 2009, 2009, Kiryu, Elsevier Science, 2010, pp. 79-83, DOI: 10.1016/j.sbspro.2010.01.017. 
[22] Product SSC-CD79P [online]. Sony, 2012 [cit. 24-01-2012]. Available at: http://www.sony. co.uk/biz/product/cctvminidomes/ssccd79p/overview

[23] POLIMENI, A., QUATTRONE, A., VITETTA, A.: A Tool for Tracing Emergency Vehicles During Evacuation, In PRATELLI, A., BREBBIA, C. A. (Eds.): Urban transport XVI: Urban transport and the environment in the $21^{\text {st }}$ century. 1st ed. Ashurst, England: WIT Press, 2010, DOI: 10.2495/UT100291.

[24] URICEK, J., POPPEOVA, V., BULEJ, V., MATIK, J.: Control and Navigation of Mobile Robots in Safety and Fire Protection. Communications - Scientific Letters of the Universtiy of Zilina, 2011, vol. 13, No. 2, pp. 118-122. ISSN 1335-4205. 